\title{
Potencialidades no Ensino-Aprendizagem da Comunicação Médico-Paciente em Três Escolas Brasileira, Espanhola e Holandesa
}

\author{
Potentialities in the Teaching-Learning of \\ Physician-Patient Communication in \\ Brazilian, Spanish and Dutch Medical Schools
}

Marcela Dohms ${ }^{1}$

Charles Dalcanale Tesser II

Suely Grosseman ${ }^{I I}$

\section{PALAVRAS-CHAVE: \\ - Relações Médico-Paciente; \\ - Comunicação em Saúde; \\ - Educação Médica.}

\section{KEYWORDS:}

- Physician-Patient Relations;

- Health Communication;

- Medical Education.

\section{Recebido em: 31/01/2012}

Reencaminhado em: 17/02/2013

Aprovado em: 20/05/2013

REVISTA BRASILEIRA DE EDUCAÇ̄̃o MÉDICA
I Prefeitura Municipal de Florianópolis, Florianópolis, SC, Brasil; Universidade Federal de Santa Catarina, Florianópolis, SC, Brasil.

"Universidade Federal de Santa Catarina, Florianópolis, SC, Brasil.

\section{RESUMO}

Está bem estabelecida a necessidade de incluir o ensino da comunicação no currículo das escolas médicas de forma sistemática. Objetivo: Conhecer a percepção de estudantes de Medicina de três escolas médicas de países diferentes (Brasil, Espanha e Holanda) e as potencialidades de cada uma destas escolas no processo de ensino-aprendizagem da comunicação médico-paciente. Método: Estudo exploratório qualitativo, com estudantes do último ano de Medicina, mediante entrevista semiestruturada, observação direta e análise temática de conteúdo. No Brasil, foram utilizados dados secundários de pesquisa similar. Resultados: As principais potencialidades encontradas foram a aprendizagem por modelos, com pacientes simulados, uso de videogravação e a Atenção Primária da Saúde (APS) como ambiente de ensino. Conclusão: A associação dessas potencialidades no ensino, com inserção do estudante na APS desde o início do curso, inclusão de pacientes simulados e videogravação, pode maximizar a aprendizagem da comunicação-médico paciente.

There is a well established requirement for the systematic inclusion of communication skills in medical school curricula. Objectives: To understand the perception of undergraduate students from three medical schools in different countries (Brazil, Spain and the Netherlands), and the potentialities of each of these schools in the teaching-learning process of physician-patient communication. Methods: Qualitative exploratory study, with final year undergraduate medical students, through semi-structured interviews and direct observation. Data analysis was conducted by means of thematic content analysis. Results: The main potentialities found were learning by models, with patient simulations, video recordings and in Primary Health Care (PHC) teaching environments. Conclusions: It was observed that the association of these teaching potentialities, with student insertion in PHC from the beginning of the course and the inclusion of simulated patients and video recordings can optimise learning of physician-patient communication. 


\section{INTRODUÇÃO}

O ensino da comunicação médico-paciente nas escolas médicas já é recomendado há mais de duas décadas em consensos e diretrizes internacionais e nacionais sobre educação médica, pois há evidências suficientes de que uma comunicação clínica adequada traz benefícios ao paciente e ao profissional, tornando mais efetivo o sistema de saúde $\mathrm{e}^{1,2,3,4,5}$.

Uma vez que as habilidades de comunicação são fundamentais à prática efetiva do cuidado ${ }^{6}$, seu ensino deve estar previsto no currículo formal, de forma sistemática, ao longo de toda a formação médica e deve abranger todas as interações que ocorrem durante o ensino-aprendizagem e de atenção à saúde, entre elas as interações entre colegas e outros profissionais envolvidos neste processo ${ }^{2,6,4}$.

Todos podem melhorar suas habilidades para conduzir um atendimento com uma comunicação adequada com pacientes, mas para isso é preciso um modelo explícito do que deve ser realizado, assim como oportunidades de prática, observações e feedback ${ }^{7,3}$.

No Brasil, desde 2001, as Diretrizes Curriculares Nacionais para os Cursos de Medicina orientam que a formação médica deve desenvolver no estudante a habilidade de se comunicar adequadamente com os pacientes e potencializar uma atitude reflexiva, ética e humanística, com capacidade para atuar em todos os níveis da atenção à saúde, visando atender às necessidades de saúde individuais e coletivas, com ênfase no SUS (Sistema Único de Saúde) $)^{8}$.

Pesquisas mostram que técnicas semelhantes, levando em conta as diferenças culturais, podem ser usadas em diferentes países nos programas de comunicação na área da saúde, pois, geralmente, as similaridades das relações de cuidado médico entre diferentes países são maiores que as diferenças ${ }^{1}$.

Entretanto, o ensino das habilidades de comunicação médico-paciente tem sido muito variado e há muito a aperfeiçoar. Para que seja realizado da melhor forma possível, é necessária a troca e a construção de conhecimentos sobre o que uma escola médica pode aprender com a outra.

Este artigo apresenta resultados de uma pesquisa que teve por objetivo analisar experiências e potencialidades de três escolas médicas em países diferentes, identificando possíveis contribuições ao desenvolvimento do ensino-aprendizagem da comunicação médico-paciente no Brasil, considerando que esse processo não ocorre de forma linear, que ensino e aprendizagem são interdependentes e que os participantes são sujeitos na busca do conhecimento de forma crítica?

Parte-se do pressuposto de que há relativo consenso entre os pesquisadores da relação médico-paciente sobre a necessidade de um conjunto de valores, conteúdos, concepções e habilidades a ser ensinado e aprendido pelos estudantes. Em consonância com os consensos internacionais sobre o tema ${ }^{10,4}$, trata-se de aumentar as relações empáticas, a capacidade de escuta, a horizontalidade das relações, o interesse pelas complexidades do universo vivencial do paciente com vistas a um cuidado integral e contextualizado, a busca de autoconhecimento pessoal e profissional permanente, uma atitude pedagógica emancipadora e empoderadora, permeada de pluralidade cultural, com vistas à construção de relações pessoais, sociais e políticas mais solidárias, comunicativas, transparentes e democratizadas ${ }^{9,11}$.

\section{METODOLOGIA}

Foi realizado um estudo exploratório qualitativo com o objetivo de analisar experiências e potencialidades no ensino-aprendizagem da comunicação médico-paciente, envolvendo três escolas médicas em países diferentes, com base nas experiências dos estudantes do último ano de Medicina. Estes foram considerados informantes privilegiados quanto ao tema, embora não o esgotem. Buscou-se compreender a percepção dos estudantes sobre sua vivência, contextualizada em seus currículos. As escolas médicas selecionadas foram as da Universidade Federal de Santa Catarina (UFSC), da Universidade Rovira i Virgili, na Espanha, e da Universidade Radboud, na Holanda.

A escola brasileira selecionada não tem um ensino de habilidades de comunicação médico-paciente estruturado e foi escolhida por conveniência e por dispor de dados sobre o tema. A seleção da escola espanhola foi devida à oportunidade de realizar a pesquisa nessa instituição, pela similaridade do Sistema de Saúde desse país com o do Brasil e pelo fato de essa escola ter inovações no ensino das habilidades de comunicação. A Holanda foi selecionada porque esse país e essa escola são uma referência importante na elaboração dos consensos internacionais sobre habilidades de comunicação e pela oportunidade de contato com profissionais e estudantes na escola estudada.

$\mathrm{Na}$ coleta de dados foram realizadas entrevistas semiestruturadas. A entrevista é bastante apropriada para a obtenção de informações sobre o que as pessoas sabem, creem, esperam, sentem ou desejam, e suas explicações ou razões a respeito delas. As entrevistas tiveram como questões norteadoras o significado da relação médico-paciente e a percepção dos estudantes sobre o ensino-aprendizagem da relação médico-paciente nas escolas.

Foram entrevistados 21 estudantes de Medicina do último ano da universidade espanhola, seguindo critérios de saturação de dados ${ }^{13}$. Na Holanda, o objetivo foi observar os diferen- 
ciais do currículo de comunicação em relação às outras duas escolas, com complementação de dados mediante entrevistas com alguns estudantes que estavam presentes nos locais de ensino visitados e entrevistas informais abertas, dirigidas com informantes locais (um docente médico de Família e Comunidade, que era o contato na instituição, e o coordenador do Departamento de Atenção Primária à Saúde). O roteiro de entrevista usado foi similar ao da Espanha. Nas escolas espanhola e holandesa, também foi empregada observação direta e estudo bibliográfico dirigido. Na escola brasileira, foram usados dados secundários de pesquisa já realizada com 25 alunos (do total de 50) do último semestre do curso de Medicina selecionados aleatoriamente por sorteio ${ }^{14}$. No tratamento dos dados foi realizada análise temática de conteúdo, com a articulação dos dados obtidos pelas diferentes estratégias de coleta, buscando uma codificação inicial, categorização e posterior junção de temas emergentes surgidos na análise das falas dos estudantes ${ }^{15}$.

O projeto de pesquisa foi aprovado pelo Comitê de Ética em Pesquisa com Seres Humanos da UFSC (Folha de Rosto número 293.503), com aprovação de adendo posterior para a coleta de dados fora do País. Na realização da entrevista, foi entregue o Termo de Consentimento Livre e Esclarecido, traduzido para o inglês e o espanhol pela pesquisadora e preenchido pelos participantes da pesquisa. Na descrição dos resultados é usada a letra B para estudantes do Brasil, E para Espanha e H para Holanda.

\section{RESULTADOS E DISCUSSÃO}

Todos os entrevistados foram unânimes sobre a importância da relação médico-paciente para uma consulta médica efetiva. Na análise dos currículos foram constatadas diferenças na ênfase dada ao ensino da comunicação médico-paciente. As três escolas médicas apresentaram diferenças e potencialidades que podem trazer contribuições ao ensino-aprendizagem da comunicação médico-paciente, as quais foram agrupadas quanto aos cenários de práticas, às estratégias e aos recursos pedagógicos.

\section{Atenção Primária à Saúde (APS) como ambiente de ensino da comunicação médico-paciente}

O contato com a realidade da comunidade por meio do estágio na APS foi uma potencialidade identificada como importante em relação ao ambiente de ensino nas três escolas. Na escola brasileira, desde o primeiro ano, os estudantes vão para a APS, tendo um contato longitudinal, regular e continuado durante todos os anos do curso. Nos outros países, mesmo a formação não sendo tão contínua, os alunos, ao entrarem em contato com a APS, valorizam esse cenário de aprendizagem. Nas três escolas, os estudantes percebem que a possibilidade do desenvolvimento de vínculos com a comunidade e da abordagem familiar favorece uma boa comunicação com o paciente. Um diferencial na escola holandesa é que as aulas teóricas dos primeiros anos são baseadas na APS. Há um Departamento de Atenção Primária na faculdade há cerca de 30 anos, e as aulas teóricas são preparadas em conjunto com as especialidades.

Na percepção dos alunos brasileiros, a vivência na APS demonstrou ser um campo de prática propício ao desenvolvimento do ensino-aprendizagem da relação médico-paciente ${ }^{16,14}$, o que vai ao encontro de outros estudos brasileiros ${ }^{17}$. Os estudantes perceberam que, quando vão precocemente à comunidade, têm mais tempo para dialogar com o paciente, favorecendo o desenvolvimento de habilidades de comunicação ${ }^{16}$. Eles relataram que a APS é o ambiente onde têm mais tempo e se sentem mais à vontade para dialogar com as pessoas, ajudando-as na superação de barreiras relacionadas à timidez inicial na comunicação ${ }^{14,30}$.

“Aprendi [a comunicação médico-paciente] principalmente no Posto de Saúde, porque é o lugar onde se tem mais tempo pra sentar e conversar, você conhece toda a família e isso deixa um ambiente mais favorável para ter uma boa relação com o paciente". (B2)

Os alunos ressaltaram a diferença entre a comunicação médico-paciente no ambiente hospitalar e na APS. A relação é considerada melhor na APS, por haver mais frequentemente demonstração de afeto, cordialidade e confiança, e relacionaram isso ao vínculo que se cria com o atendimento longitudinal, com acompanhamento do mesmo grupo de pessoas por longo tempo. No atendimento hospitalar, referiram que os atendimentos são mais breves, pontuais, não propiciando maior aproximação com os pacientes, e os observaram como mais distantes.

"Na APS, as pessoas estão há muitos anos com o mesmo médico e parece que há muito mais amizade, $e$ por assim dizer carinho, de uma certa maneira". (E7)

Perceberam que quanto mais conhecem o paciente, sua família e o seu contexto, mais fácil é ter uma boa relação médico-paciente:

"Aqui [na APS] você conhece as pessoas há mais tempo, de maneira diferente, conhece a família, como eles vivem, problemas em casa e sabe mais sobre os pacientes. Aqui [APS], frequentemente a comunicação é 
melhor que no hospital... Eles [os médicos de família] conhecem muito bem o paciente, e o paciente conhece o médico há dez anos, por exemplo, se tem filhos, como as crianças vão indo... é mais familiar, mais uma unidade, não é só uma pessoa com dor de cabeça, como às vezes num neurologista. É por isso que eu acho que a APS é diferente do hospital, onde geralmente é mais distante. Eu acho que aqui é melhor porque se conhece todos ao redor e você sabe mais sobre as pessoas". (H1)

Alguns alunos notaram que no hospital é mais comum os médicos fornecerem menos informações aos pacientes do que na APS:

“Uma reclamação que os pacientes sempre fazem no hospital é que lhes falta informação. É que ninguém lhes diz nada. Em Atenção Primária isso nunca ocorreu. No hospital perguntam: 'Você que está com o médico? Pode me dizer o que está acontecendo?"'. (E2)

Essas constatações são previsíveis, uma vez que a APS é um espaço privilegiado para o ensino-aprendizagem da comunicação médico-paciente devido aos seus atributos de local de cuidado clínico longitudinal e de coordenação desse cuidado, independentemente da natureza dos problemas de saúde, de modo interdisciplinar e integrando promoção de saúde e prevenção de doenças com o cuidado clínico ao adoecimento, de modo acessível, com adequação cultural, abordagem familiar e comunitária ${ }^{18}$.

A Atenção Primária facilita as relações entre pacientes e médicos, os pacientes participam da tomada de decisões sobre sua saúde, além da criação de vínculos entre a atenção de saúde individual e as famílias e comunidades dos pacientes (OMS, 2008).

Conforme Starfield ${ }^{18}$, a APS no Brasil é o locus de cuidado estratégico do Sistema Único de Saúde para a sua completa constituição e implantação, tornando-se um cenário prioritário de ensino e pesquisa em habilidades de comunicação. A APS é o primeiro ponto de contato com o sistema de saúde, é a primeira tentativa de um profissional entender os pacientes e ajudá-los a entender seus sintomas e a melhorar a própria capacidade de lidar com os problemas, o que geralmente não requer muita tecnologia médica dura ${ }^{7,19}$.

Além disso, é difícil entender o paciente sem ter conhecimento da sua vida e trabalho e de seu contexto cultural e social $^{7}$. Assim, a vivência de um estudante na APS traz a possibilidade de conhecer a pessoa num sentido amplo, aprender um cuidado integral e longitudinal por meio do conhecimento da realidade e modo de vida das pessoas que vivem em determinada comunidade.

Reiterando esse achado, um estudo de coorte histórica, realizado no Canadá, comparando uma escola com ensino baseado em problemas da comunidade com outras três escolas de ensino tradicional, mostrou que a transição para um ensino baseado em problemas da comunidade foi associado com a melhora do cuidado integral, sem declínio no diagnóstico e manejo. Os médicos graduados em escolas médicas tradicionais no mesmo período não exibiram a mesma melhora no desempenho ${ }^{20}$.

Starfield ${ }^{18}$ concluiu em seus estudos que é necessária a reestruturação dos sistemas de saúde e das interações profissional-paciente para trazê-los para uma relação mais próxima com outros sistemas sociais, no contexto da atenção à saúde de populações, para tornar a atenção médica mais efetiva e equitativa na prevenção, cuidado e cura das doenças e na promoção da saúde.

Assim, práticas no contexto da APS durante todo o curso se têm demonstrado muito importantes para a formação médica mais voltada às necessidades reais da população. É na ida à comunidade na APS que o aluno tem a possibilidade de aprender com a realidade, sair das fronteiras da universidade e do hospital, entrar na vida das pessoas e conhecer seu contexto sociocultural, o que é fundamental para o entendimento do paciente ${ }^{7,21}$.

Alguns estudantes observaram que essas diferenças são favorecidas pelo ambiente, mas não necessariamente determinadas por ele. Entendem que a APS pode favorecer o desenvolvimento de modelos positivos de comunicação médico-paciente, mas as características dos profissionais que os supervisionam interferem diretamente no tipo de relação clínica, que pode ser positivo ou negativo, tanto na APS como no hospital. Esses estudantes realçaram a potência do ensino por meio de modelos em todos os cenários, como pode ser visto a seguir.

\section{Aprendizagem da comunicação médico-paciente por modelos}

Os estudantes de todas as escolas mencionaram a importância dos modelos para a aprendizagem da relação médico-paciente. Enquanto as atitudes consideradas adequadas são incorporadas pelos estudantes, as negativas são identificadas como atitudes que devem ser evitadas ${ }^{14,30}$ :

"Principalmente no dia a dia, quando a gente vê uma situação e então pensa: 'não, não é assim que eu gostaria de ser" ou "é assim que eu gostaria de conduzir"'. (B13) 
Na escola holandesa, foi relatado que a maioria dos professores atua como modelo positivo na comunicação médico-paciente e que, quando há um modelo negativo, este é facilmente identificado e criticado.

"Por exemplo, se vemos um médico tratando uma pessoa idosa de maneira rude, comentamos entre nós que não deveria tratar assim. Mas isso não é muito comum, vemos mais com os médicos antigos".

Talvez isso ocorra pelo diferencial derivado do ensino de um currículo de comunicação bem estruturado ao longo do curso. Modelos podem ter um profundo efeito na aprendizagem de atitudes ${ }^{22,23}$. Na medicina, a aprendizagem por modelos foi provavelmente a mais importante, senão única, forma geral de aprendizado, antes da institucionalização do ensino médico nas academias, que o separou do ambiente de prática social do cuidado médico ${ }^{24}$.

No estudo realizado na escola brasileira, Grosseman e Stoll ${ }^{16}$ relatam que as interações entre docente e discente que ocorrem no processo de ensino médico transmitem mensagens, muitas vezes, ambíguas, referentes ao relacionamento médico-paciente.

Sabe-se que, além do currículo formal, normas e valores diversos são veiculados por diferentes atores sociais que entram em contato com o estudante, o que constitui um currículo oculto ${ }^{25,26}$. A maneira como o professor ensina e se relaciona com os estudantes já é um modo de formação. O tipo de interação entre professor e estudante influencia o aprendizado, e por isso é importante a construção e o exercício de relações de parceria. Nesse sentido, o ensino centrado no aprendiz reforça e induz o ensino da consulta centrada no paciente, o que melhora a comunicação. A tomada de decisão compartilhada na consulta precisa ter modelos no relacionamento entre professor e aprendiz. A responsabilidade do professor é demonstrar na prática cotidiana as lições que deseja que o aluno aprenda ${ }^{7}$.

Inconsistências entre o que se aprende na sala de aula e o que se vê na prática, com mensagens divergentes, podem levar os estudantes a desvalorizar a relação médico-paciente e a concluir que as habilidades de comunicação não são relevantes no seu cuidado ${ }^{16,27}$.

A possibilidade e a proliferação de bons modelos para os alunos estão relacionadas ao ambiente do ensino-aprendizagem. O ambiente hospitalar - especialmente das enfermarias - parece não ser o mais propício como único recurso para o ensino-aprendizagem da comunicação médico-paciente. Isto é compreensível devido à subcultura do ambiente hospitalar, mais autoritária, em razão do alto grau de controle aceito e legitimado que os médicos exercem sobre os doentes, e devido às características dos adoecimentos ali presentes ${ }^{28}$. Os ambientes ambulatoriais, e particularmente a APS, como antes discutido, foram relatados como ambientes que facilitam a oferta de bons modelos.

Segundo Kuhn ${ }^{29,30}$, a aprendizagem por semelhança é também uma forma fundamental de iniciação na atividade científica, inculcadora das práticas de resolução de problemas de qualquer paradigma científico. Na acepção de Fleck ${ }^{31}$, a conformação da percepção e das práticas na iniciação em um estilo de pensamento ocorre da mesma forma. A atividade clínica não é uma atividade científica, mas é cada vez mais influenciada por ela, e o uso do termo paradigma foi estendido à medicina. Assim, fala-se com propriedade em paradigma flexneriano e seus problemas ${ }^{24}$, muito vinculado ao ambiente hospitalar, e compreende-se por que a APS tem se mostrado um ambiente necessário e propício ao ensino ${ }^{42}$. Logo, a oferta de bons modelos a serem inspiradores dos estudantes é uma estratégia fundamental de ensino-aprendizagem da comunicação médico-paciente (assim como de outras habilidades clínicas).

\section{Aprendizagem da comunicação médico-paciente por meio de pacientes simulados}

Como estratégias de ensino estruturadas, na escola brasileira estudada foram relatadas apenas aulas teóricas pontuais e algumas aulas práticas de Semiologia ${ }^{14}$. Na escola espanhola, além de aulas teóricas, há treinamento com pacientes simulados instrutores, usados também na Holanda.

A prática com pacientes simulados que fornecem feedback após a simulação foi enfatizada pela maioria dos alunos entrevistados na escola espanhola. Esse treinamento é realizado nos três últimos anos, no Laboratório de Comunicação, e relatado como importante para o aprendizado da comunicação médico-paciente:

"Temos isso dos pacientes simulados, que ajudam muitíssimo, te ensinam o que melhorar, o que fazes bem e o que fazes mall...] E temos a sorte de começar no terceiro ano e então no sexto estamos tranqüilos". (E5)

Alguns alunos entenderam que o treinamento com simulação é essencial e que

"ajuda muito receber um feedback de como alguém te vê como médico" (E7).

Disseram que gostam de saber o que estão fazendo bem e de receber sugestões de como podem melhorar suas habili- 
dades de comunicação. Perceberam que com o treinamento se sentem progressivamente mais seguros para se relacionar com os pacientes. Os pacientes simulados nessa escola são voluntários, pessoas treinadas para isso e que recebem um valor pelas horas de trabalho.

Na escola holandesa, também existe o treino de habilidades de comunicação com pacientes simulados, que ocorre a cada novo módulo, em cada incorporação de uma nova disciplina, transversalmente a todo o currículo. Por exemplo, ao iniciarem Neurologia, os estudantes treinam o exame neurológico com pacientes simulados e já trabalham a comunicação junto. Há um tutor, médico de família ou psicólogo, que observa e dá feedback em grupos pequenos, ou pode ser o próprio paciente quem dá o feedback individualmente.

Alunos da escola holandesa consideraram que os pacientes simulados ajudam a aprender a fazer perguntas que facilitem a fala do paciente, auxiliam o aluno a ser mais empático e a melhorar a relação de confiança:

"Você tem que fazer a pergunta certa para ter mais informações e também aprender não só o que você tem que perguntar, mas perguntar bem, com as palavras mais empáticas, para que o paciente confie em você, no que você está explicando". (H1)

Facilita muito praticar uma situação em simulação antes de enfrentá-la diretamente com o paciente. Foi relatado que a reflexão em pequenos grupos estimula a autopercepção. Os pacientes simulados auxiliam em como fazer os pacientes perceberem que estão sendo ouvidos, em como perguntar da melhor maneira, praticando o uso de questões abertas:

"Treinamos como fazer os pacientes saberem que estamos ouvindo, que tipo de questão perguntar, o mais aberta possível, e como fazê-los perceber que você pode se relacionar com os problemas deles [também nos aspectos sociais]". (H2)

Os estudantes valorizaram poder praticar a simulação a cada incorporação de conteúdos para melhorar suas habilidades de comunicação. Percebem que os pacientes simulados ajudam a conseguir relacionar os problemas físicos dos pacientes com os problemas sociais e fazê-los perceber essas relações:

"A maior parte do tempo temos pacientes simulados. Eles ensinam a refletir sobre como você fez. Eisto éa coisa mais importante, claro, é como aprendemos." (H1).
Ressaltaram que é especialmente importante para aprender como dar más notícias ao paciente e como isso se reflete em suas próprias emoções.

Pacientes simulados têm sido usados com sucesso no ensino de comunicação desde a década de 1960 1,32. Algumas escolas no Brasil também já os utilizam. A simulação de pacientes oferece a oportunidade de experimentar e aprender em um ambiente seguro, sem a possibilidade de prejudicar pacientes reais. As simulações podem ser padronizadas, com reprodução dos mesmos papéis específicos para todos os estudantes, improvisadas ou personalizadas de acordo com a dificuldade individual. Possibilitam ainda refazer uma situação de dificuldade. Podem ocorrer em situações programadas ou entre pacientes reais. Pesquisas mostraram que estudantes, residentes e médicos não conseguem distinguir pacientes reais de pacientes simulados bem treinados ${ }^{1}$.

Além disso, o paciente pode ser treinado a fornecer feedback a respeito da comunicação ${ }^{1}$ :

"Os pacientes simulados podem nos dar feedback em como se sentiram com a maneira que fizemos" (H2).

Esse sistema de ensino de simulação de pacientes proporciona excelente treinamento para os alunos identificarem e refletirem sobre as especificidades da relação com os pacientes $^{33}$, até mesmo para a prática da longitudinalidade ${ }^{34}$. Kurtz ${ }^{1}$, baseado em vários estudos, concluiu que seu uso é efetivo, confiável e aceito pelos estudantes.

\section{Aprendizagem da comunicação médico-paciente por videogravação com feedback}

O recurso da videogravação com feedback como estratégia para o ensino sistematizado, além do uso de pacientes simulados, ajuda muito no aprendizado da comunicação médico-paciente, segundo a percepção dos alunos da escola médica holandesa. Eles relataram que o feedback do tutor e dos colegas facilita lidar com as situações cotidianas e que participar de um grupo assistindo um colega ajuda no próprio aprendizado:

"Com videocâmera, você depois se vê e reconhece que tem às vezes uma má relação médico-paciente [...] Para mim é importante aprender como eu posso fazer isso melhor [...] É muito importante reconhecer que você fez algo[...]" (H1)

Informaram que a videogravação pode ser de simulações periódicas, que são posteriormente revistas junto com um psicólogo ou médico e outros estudantes em um grupo pequeno, 
para discutir o que foi bom ou ruim e como lidar melhor com essas situações.

Sabe-se que, além de conteúdos teóricos, é preciso que ocorra na prática a observação de consultas realizadas pelos alunos, tanto diretamente como por videogravações. As filmagens permitem que os alunos revejam seus comportamentos e atitudes, possibilitando a discussão do significado de cada postura assumida ${ }^{33}$. Maguire $^{32}$ encontrou superioridade evidente nos profissionais treinados com a técnica de videofeedback interativo frente à técnica convencional. Vários trabalhos posteriores têm confirmado a importância dessa técnica para o sucesso no ensino das habilidades de comunicação ${ }^{35}$.

A auto-observação possibilita ver a si mesmo, ver o que exatamente se está fazendo e onde é possível melhorar. E o uso do vídeo com feedback por outras pessoas oferece muitas vantagens em relação à observação solo ${ }^{36}$. Além disso, a gravação em vídeo possibilita a observação e o feedback da comunicação não verbal, que são perdidos em outras modalidades de ensino. Não há dúvida de que o uso de gravação em vídeo representa o padrão-ouro no ensino de comunicação ${ }^{1}$.

Apesar das evidências favoráveis, poucos programas de treinamento para a entrevista médica com esse formato têm sido implementados no Brasil e em outros países. Isto tem sido creditado, parcialmente, ao fato de esse formato ser considerado estressante por alunos e professores ${ }^{37}$. Entretanto, em países como Reino Unido e Holanda, a maioria das escolas médicas usa sistematicamente uma combinação de ensino em pequenos grupos, dramatizações com pacientes simulados e feedback com gravações em vídeo, com resultados satisfató$\operatorname{rios}^{7}$, seguindo as recomendações do último consenso inglês a respeito do currículo de comunicação na graduação médica ${ }^{4}$.

No Brasil, há algumas experiências com a técnica de Problem Based Interview ${ }^{38}$, com residentes de Medicina de Família e como educação permanente para um grupo de médicos da APS em Florianópolis ${ }^{39}$. Também há experiências com simulação e videogravação na graduação de Medicina e em outras residências médicas ${ }^{40}$, mas não há uma sistematização para todas as universidades.

As escolas brasileiras, no geral, não têm um ensino estruturado, apresentam iniciativas ainda tímidas e precisam estruturar melhor suas estratégias. Nesse sentido, muito podemos aprender através da percepção dos estudantes e com o exemplo de experiências internacionais, como as citadas da Holanda e Espanha.

Embora esta pesquisa se limite a três escolas, sendo os dados do Brasil reutilizados, seus resultados sugerem a necessidade de novas pesquisas, incluindo mais escolas e experiências que permitam melhores comparações e aprofunda- mentos, de modo a reforçar mobilizações nas escolas médicas brasileiras para um melhor ensino-aprendizagem da comunicação médico-paciente.

Por fim, Sucupira ${ }^{33}$ ressalta a importância de considerar no ensino das habilidades de comunicação todos os determinantes envolvidos na relação e alerta para o risco de se reduzir o caráter da relação clínica a uma simples técnica ou procedimento aprendido por protocolos caso não se considerem fatores como o contexto em que ela ocorre e a inserção social dos atores envolvidos.

\section{CONCLUSÃO}

Há muitos pontos em comum em relação ao ensino-aprendizagem da comunicação médico-paciente. Cada escola estudada tem potencialidades que podem contribuir para a melhora do ensino das habilidades de comunicação em outras escolas.

Assim como o aprendizado por modelos, forma natural de apreensão das atitudes a serem assumidas na relação médico-paciente, a imersão no contexto da APS ao longo do currículo contribui para o aprendizado da interação médico-paciente e com a equipe interdisciplinar. O ensino sistemático de habilidades de comunicação também deve ser inserido para que se potencialize o ensino-aprendizado da relação. É necessário, ainda, ensinar como lidar com pacientes e suas famílias em situações sensíveis, em diversos contextos e faixas etárias, em práticas supervisionadas mediante observações e feedback, em simulações ou videogravações, como observado na Espanha e Holanda.

No Brasil, apesar de algumas escolas apresentarem ganhos importantes no tema ${ }^{41,35,40}$, há poucas publicações, comparativamente à literatura internacional. Embora várias escolas tenham pacientes simulados, ainda há escolas no Brasil, como a investigada, em que não há um conteúdo de comunicação inserido no currículo formal.

As diretrizes curriculares relacionadas à comunicação médico-paciente, apesar de significarem um avanço importante para a educação médica brasileira, ainda não são seguidas de maneira sistemática e se dão predominantemente por modelos na escola brasileira aqui estudada e em outras escolas médicas brasileiras. O desafio é que todas as escolas médicas tenham um programa curricular de comunicação médico-paciente que possa unir todas essas potencialidades: o estudante exposto desde o primeiro ano ao trabalho interdisciplinar e na comunidade, o ensino sistematizado da comunicação médico-paciente através de treinamento com pacientes simulados e videogravação, com flexibilidade para se adaptar às necessidades de cada estudante.

É importante que os formadores tenham a oportunidade de aperfeiçoamento nas habilidades de comunicação para que possam facilitar a aprendizagem. É necessário também criar 
programas de desenvolvimento para preparar os docentes para o ensino-aprendizado das habilidades de comunicação em todos os níveis de formação, sensibilizar diretores de programas e administradores acerca da importância de desenvolver excelentes programas de comunicação em suas instituições e aumentar a formação de facilitadores e professores neste tema tão importante para a formação de bons médicos.

Por último, é fundamental que o ensino de habilidades de comunicação não seja visto apenas como técnicas a serem aplicadas, mas, sim, como possibilidades de melhorar a comunicação significativamente, levando em conta os diversos determinantes da situação e do processo saúde-doença do paciente, assim como o ambiente em que a comunicação ocorre. Para o estudante, a forma como é tratado pelo professor é um dos primeiros processos de aprender a se relacionar. Valores como cidadania e respeito permeiam (ou não) as relações interpessoais. Assim, deve-se buscar a construção de um ambiente de ensino com relacionamentos dialógicos e reflexivos com o professor, com a comunidade e com a equipe. Também há que ter sensibilidade para se relacionar de forma interdisciplinar e resistir às pressões laborais que podem induzir a indiferença e o distanciamento humano e afetivo. Tarefas complexas, mas de grande potencial para a melhoria das relações na formação médica.

\section{AGRADECIMENTO}

O autor Charles D. Tesser agradece ao CNPq o subsídio financeiro (Processo n ${ }^{\circ}$ 311193/2010-2).

\section{REFERENCIAS}

1. Kurtz S, Silverman J, Draper J. Teaching and learning communication skills in medicine. $2^{\text {nd }}$ ed. Oxford, UK: Radcliffe Medical Press;2005.

2. Simpson M, Buckman R, Stewart M, Maguire P. Lipkin M, Novack D, Till J. Doctor-patient communication: the Toronto consensus statement. BMJ. 1991;303(6814):1385-7. DOI: $10.1136 /$ bmj.303.6814.1385.

3. General Medical Council. Good Medical Practice. London: GMC;2001.

4. Von Fragstein M, Silverman J, Cushing A, Quilligan S, Salisbury H, Wiskin C. UK consensus statement on the content of communication curricula in undergraduate medical education. Med Educ. 2088;42(11):1100-7. DOI: 10.1111/j.1365-2923.2008.03137.x

5. Dent J, Harden RM, editors. A practical guide for medical teachers. $3^{\text {th }}$ ed. Toronto: Elsevier;2009.

6. Makoul G. Essential elements of communication in medical encounters: the kalamazoo consensus statement. Acad Med. 2001;76(4):390-3. DOI: 10.1097/00001888-200104000-00021
7. Pendleton D, Schofield T, Tate P, Havelock P. The new Consultation: developing doctor-patient communication. Oxford; 2007.

8. Brasil. Ministério da Educação. Conselho Nacional de Educação. Câmara de Educação Superior. Resolução CNE/CES n. ${ }^{\circ}$ 4, de 7 de novembro de 2001. Institui diretrizes curriculares nacionais do curso de graduação em medicina. [Internet]. Brasília, 2001; [acesso em 18 out. 2009]. Disponível em: http://portal.mec.gov.br/cne/arquivos/ pdf/CES04.pdf

9. Freire P. Pedagogia da autonomia: Saberes necessários à prática educativa. São Paulo: Paz e Terra;1996.

10. Association of American Medical College. Report 1: Learning objectives for medical student education:Guidelines for medical Schools. Washington: AAMC;1998.

11. Ayres JRCM. Cuidado e reconstrução das práticas de Saúde. Interface Comun. Saúde Educ. 2004;8(14): 73-92. DOI: 10.1590/S1414-32832004000100005.

12. Minayo MCS. O desafio do conhecimento: pesquisa qualitativa em saúde. 8ae ed. São Paulo: Hucitec/ABRASCO; 2004.

13. Fontanella BJB, Ricas J, Turato ER. Amostragem por saturação em pesquisas qualitativas em saúde: contribuições teóricas. Cad Saúde Pública. 2008;24(1):17-27. DOI: 10.1590/S0102-311X2008000100003

14. Stock F. Percepção de estudantes de medicina da Universidade Federal de Santa Catarina sobre o ensino-aprendizagem da relação médico-paciente antes e depois da reforma curricular. Santa Catarina; 2009. Graduação [Trabalho de conclusão do curso] — Universidade Federal de Santa Catarina.

15. Bardin L. Análise de conteúdo. Trad. Luís Antero Reto e Augusto Pinheiro. Lisboa: Edições 70;2002.

16. Grosseman S, Stoll C. O ensino-aprendizagem da relação médico-paciente. Estudo de caso com estudantes do último semestre do curso de medicina. Rev Bras Educ Med. 2008;32(3):301-8. DOI: 10.1590/S0100-55022008000300004

17. Ferreira RC, Fiorini VML, Crivelaro E. Formação profissional no SUS: o papel da atenção básica em saúde na perspectiva docente. Rev Bras Educ Med. 2010;34(2):207-15. DOI: 10.1590/S0100-55022010000200004

18. Starfield B. Atenção Primária, equilíbrio entre necessidades de saúde, serviços-tecnologia. Brasília: UNESCO/Ministério da Saúde;2004.

19. Merhy EE, Franco TB. Por uma Composição Técnica do Trabalho Centrada nas Tecnologias Leves e no Campo Relacional. Saúde Debate. 2003;27(65):316-323.

20. Tamblyn R, Abrahamowicz M, Duphinee D, Girard N, Bartlett G, Grand'Maison P, Brailovsky C. Effect of a commu- 
nity oriented problem based learning curriculum on quality of primary care delivered by graduates: historical cohor comparison study. BMJ. 2005;331:1002. DOI: 10.1136/ bmj.38636.582546.7C .

21. Worley P, Prideaux D, Strasser R, Magarey A, March R. Empirical evidence for symbiotic medical education: a comparative analysis of community and tertiary-based programmes. Med Educ. 2006;40(2):109-16.

22. Siegler M, Reaven N, Lipinski R, Stocking C. Effect of role-model clinicians on student's attitudes in a second-year course on introduction to the patient. Fam Med. 1987;28.

23. Ficklin FL, Browne VL, Powell RC, Carter JE. Faculty and house staff members as role models. J Med Educ. 1988;63(5):392-6.

24. Lampert JB. Tendências de mudança na formação médica no Brasil: tipologia das escolas. 2ª ed. São Paulo: Hucitec; 2009.

25. Hafferty FW, Franks R. The hidden curriculum, ethics teaching, and the structure of medical education. Acad Med. 1994;69(11):861-71.

26. Lempp H, Seale C. The hidden curriculum in undergraduate medical education: qualitative study of medical students' perceptions of teaching. BMJ. 2004;329(7469):770-3. DOI: $10.1136 /$ bmj.329.7469.770

27. Haidet P, Stein HF. The role of the student-teacher relationship in the formation of physicians. J Gen Intern Med. 2006;21(Suppl.1):S16-S20. DOI: 10.1111/j.1525$-1497.2006 .00304 . x$.

28. Tesser CD. Três considerações sobre a "má medicina". Interface Comun. Saúde Educ. 2009;13(31):273-86. DOI: 10.1590/S1414-32832009000400004

29. Kuhn TS. A estrutura das revoluções científicas. São Paulo: Perspectiva; 1987.

30. Kuhn, T.S. A tensão essencial. Lisboa: Edições 70, 1989.

31. Fleck L. Gênese e desenvolvimento de um fato científico. Belo Horizonte: Fabrefactun; 2010.

32. Maguire P. The use of patient simulation in training medical students in history-talking skills. Med Biol Illus. 1976;26.

33. Sucupira AC. A importância do Ensino da relação médico-paciente e das habilidades de comunicação na formação do profissional de saúde. Interface Comun. Saúde Educ. 2007. 11(23):624-7. DOI: 10.1590/S1414-32832007000300016

34. Linssen T, Van Dalen J, Retnans JJ. Simulating the longitudinal doctor-patient relationship: experiences of simulated patients in successive consultations. Med Educ. 2007;41(9): 873-8. DOI: 10.1111/j.1365-2923.2007.02850.x

35. De Marco MA, Vessoni AL, Capelo A, Dias CC.. Laboratório de comunicação: ampliando as habilidades do estudante de medicina para a prática da entrevista. Interface
Comun. Saúde Educ. 2010;14(32):217-27. DOI: 10.1590/ S1414-32832010000100018.

36. Beckman HB, Frankel RM. The use of videotape in internal medicine training. J Gen Intern Med. 1994;9(9):517-21. DOI: $10.1007 /$ BF02599224.

37. Nilsen S, Baerheim A. Feedback on video recorded consultations in medical teaching: why students loathe and love it - a focus-group based qualitative study. BMC Med Educ. 2005;5:28. DOI: 10.1186/1472-6920-5-28.

38. Lesser AL. Problem-Based interviewing in general practice: a model. Med Educ. 1985;19(4):299-304. DOI: 10.1111/ j.1365-2923.1985.tb01325.x

39. Borrel F, Dohms MC, Fontcuberta JMB. Utilização da filmagem de consultas para o aprendizado. In: Gusso GD, Lopes JM. Tratado de Medicina de Família e Comunidade. Porto Alegre: Artmed; 2012.

40. Ballester D. Ensino do residente de pediatria em um ambulatório geral : análise da consulta. São Paulo; 2009. Doutorado [Tese] — Faculdade de Medicina da Universidade de São Paulo.

41. Turini B, M Neto D, Tavares MS, Nunes SOV, Silva VLM, Thomson Z. Comunicação no ensino médico: estruturação, experiência e desafios em novos currículos médicos. Rev Bras Educ Med. 2008;32(2):264-70. DOI: 10.1590/S010055022008000200015.

42. Tesser CD. Contribuições da Epistemologia de Kuhn e Fleck para reforma do ensino médico. Rev Bras Educ Med. 2008;32(1):98-104. DOI: 10.1590/S0100-55022008000100013.

\section{CONTRIBUIÇÃO DOS AUTORES}

Os três autores participaram de todas as fases da pesquisa, incluindo concepção, planejamento, discussão dos achados e elaboração do artigo, incluindo a revisão e aprovação da versão final, excetuando-se a coleta de dados, realizada por Marcela Dohms..

\section{CONFLITO DE INTERESSES}

Declarou não haver.

\section{ENDEREÇO PARA CORRESPONDÊNCIA}

Marcela Dohms

Rua Professor Brandão, 178, apto. 305.

Curitiba

CEP 80040-010 PR 